\title{
Boundedness of Solutions for Second Order Differential Equations
}

\author{
Daniel C. Biles
}

Correspondence: Department of Mathematics and Computer Science, Belmont University, Nashville, Tennessee, USA

Received: June 6, 2020 Accepted: July 1, 2020 Online Published: July 10, 2020

doi:10.5539/jmr.v12n4p58 URL: https://doi.org/10.5539/jmr.v12n4p58

\begin{abstract}
We present new theorems which specify sufficient conditions for the boundedness of all solutions for second order nonlinear differential equations. Unboundedness of solutions is also considered.
\end{abstract}

Keywords: asymptotic behavior, boundedness, nonlinear differential equation, second order differential equation

\section{Introduction}

This article was originally motivated by Tunç and Ayhan (Tunç \& Ayhan, 2015) (that generalized a paper by Qarawani (Qarawani, 2012) and used a similar proof) which, after a substitution, specified conditions for the boundedness of solutions of the differential equation

$$
y^{\prime \prime}(x)-y^{\prime}(x)+y(x)=e^{2 x} h\left(e^{x}\right)[y(x)]^{\alpha}
$$

where $\alpha \in(-1,0) \cup(0,1)$. The proof of this result is especially attractive, since it only involves simple integral inequalities. We will discuss this method of proof further later in the article. Our goal is to generalize this result - for example, it seems that it should be possible to generalize the right-hand side quite a bit. The desired generalization is Theorem 1 below. Following Theorem 1, we investigate this problem further and prove some related facts and also results for additional cases.

Definition 1 The function $y$ is called a solution of

$$
y^{\prime \prime}(x)+p(x) y^{\prime}(x)+q(x) y(x)=h(x) f(y(x)) \text { for } x \geq x_{0}
$$

on $\left[x_{0}, \infty\right)$ if $y$ and $y^{\prime}$ are absolutely continuous on compact subintervals of $\left[x_{0}, \infty\right)$ and $y$ satisfies the differential equation a.e. on $\left[x_{0}, \infty\right)$.

We shall call a solution $y$ positive if $y>0$ on $\left(x_{0}, \infty\right)$.

\section{Results}

Theorem 1 Consider the differential equation

$$
y^{\prime \prime}(x)+p(x) y^{\prime}(x)+q(x) y(x)=h(x) f(y(x)) \text { for } x \geq x_{0} .
$$

Assume

1) $p$ is absolutely continuous on compact subintervals of $\left[x_{0}, \infty\right)$ and $p \geq 0$ on $\left[x_{0}, \infty\right)$,

2) $q$ is absolutely continuous on compact subintervals of $\left[x_{0}, \infty\right)$ and there exists a $q_{0} \in \boldsymbol{R}$ such that $q(x) \geq q_{0}>0$ for all $x \in\left[x_{0}, \infty\right)$,

3) $q^{\prime} \leq 0$ a.e. on $\left[x_{0}, \infty\right)$,

4) $h$ is absolutely continuous on compact subintervals of $\left[x_{0}, \infty\right)$,

5) $\int_{x_{0}}^{\infty}\left|h^{\prime}(s)\right| d s<\infty$,

6) There exists an $r \in(-1,1)$ and a $c \in(0, \infty)$ such that $0<|f(w)| \leq c|w|^{r}$ for all $w \neq 0$,

7) For all $w \in \mathbf{R}, F(w) \equiv \int_{0}^{w} f(u) d u$ exists and is finite.

Then, any solution of (1) on $\left[x_{0}, \infty\right)$ is bounded. 
Note 1. If we know that a solution is nonnegative, we only need assumptions 6 and 7 to hold for $w>0$. (Similarly in the case of a negative solution.) For example, in the case $f(w)=w^{\frac{1}{2}}$, it is clear any solution will remain nonnegative, and this choice of $f$ satisfies assumptions 6 and 7 for $w>0$. Otherwise these assumptions must hold for all $w \neq 0$ to allow a solution to change sign. We note that this also holds for results which occur later in the article.

Proof. We begin by multiplying both sides of (1) by $y^{\prime}$ and integrating from $x_{0}$ to $x$, where $x_{0}<x$ :

$$
\int_{x_{0}}^{x} y^{\prime \prime}(s) y^{\prime}(s) d s+\int_{x_{0}}^{x} p(s)\left[y^{\prime}(s)\right]^{2} d s+\int_{x_{0}}^{x} q(s) y(s) y^{\prime}(s) d s=\int_{x_{0}}^{x} h(s) f(y(s)) y^{\prime}(s) d s .
$$

If we apply integration by parts to the third term on the left-hand side we obtain

$$
\left(q(x)[y(x)]^{2}\right) / 2-\left(q\left(x_{0}\right)\left[y\left(x_{0}\right)\right]^{2}\right) / 2-(1 / 2) \int_{x_{0}}^{x} q^{\prime}(s)[y(s)]^{2} d s .
$$

Applying integration by parts to the term on the right-hand side, we obtain

$$
h(x) F(y(x))-h\left(x_{0}\right) F\left(y\left(x_{0}\right)\right)-\int_{x_{0}}^{x} h^{\prime}(s) F(y(s)) d s .
$$

Substituting these two expressions into (2), we have

$$
\begin{gathered}
\left(\left[y^{\prime}(x]^{2}\right) / 2-\left(\left[y^{\prime}\left(x_{0}\right]^{2} / 2\right)+\int_{x_{0}}^{x} p(s)\left[y^{\prime}(s)\right]^{2} d s+\left(q(x)[y(x)]^{2}\right) / 2-\left(q\left(x_{0}\right)\left[y\left(x_{0}\right)\right]^{2}\right) / 2-\right.\right. \\
(1 / 2) \int_{x_{0}}^{x} q^{\prime}(s)[y(s)]^{2} d s=h(x) F(y(x))-h\left(x_{0}\right) F\left(y\left(x_{0}\right)\right)-\int_{x_{0}}^{x} h^{\prime}(s) F(y(s)) d s .
\end{gathered}
$$

Letting $K=\left[y^{\prime}\left(x_{0}\right]^{2}+q\left(x_{0}\right)\left[y\left(x_{0}\right)\right]^{2}-2 h\left(x_{0}\right) F\left(y\left(x_{0}\right)\right)\right.$ yields

$$
\begin{gathered}
{\left[y^{\prime}(x)\right]^{2}+2 \int_{x_{0}}^{x} p(s)\left[y^{\prime}(s)\right]^{2} d s+\left(q(x)[y(x)]^{2}\right)-\int_{x_{0}}^{x} q^{\prime}(s)[y(s)]^{2} d s=2 h(x) F(y(x))-} \\
2 \int_{x_{0}}^{x} h^{\prime}(s) F(y(s)) d s+K .
\end{gathered}
$$

Applying assumptions 1 and 3, we see that the terms $\left[y^{\prime}(x]^{2}, 2 \int_{x_{0}}^{x} p(s)\left[y^{\prime}(s)\right]^{2} d s\right.$ and $-\int_{x_{0}}^{x} q^{\prime}(s)[y(s)]^{2} d s$ are each nonnegative, and also applying assumption 2, we obtain

$$
q_{0}[y(x)]^{2} \leq 2|h(x)||F(y(x))|+2 \int_{x_{0}}^{x}\left|h^{\prime}(s)\right||F(y(s))| d s+|K|
$$

It follows from assumption 5 that $|h|$ is bounded by some $H \geq 0$ on $\left[x_{0}, \infty\right)$. We then have

$$
[y(x)]^{2} \leq 2 H|F(y(x))| / q_{0}+\left(2 / q_{0}\right) \int_{x_{0}}^{x}\left|h^{\prime}(s)\right||F(y(s))| d s+|K| / q_{0} .
$$

Define $M:\left[x_{0}, \infty\right) \rightarrow \boldsymbol{R}$ by $M(x)=\max _{s \in\left[x_{0}, x\right]}|y(s)|$. Without loss of generality, we may assume there is a positive $k \in \boldsymbol{R}$ such that $M(x) \geq k$ for all $x \geq x_{0}$ (we continue to use $x_{0}$ for convenience). Note from assumption 6 that $|F(y(s))| \leq c[M(x)]^{r+1} /(r+1)$ for all $s \in\left[x_{0}, x\right]$. Applying this to (4) we have

$$
[M(x)]^{2} \leq 2 H c[M(x)]^{r+1} /\left[q_{0}(r+1)\right]+\left(\left[2 c[M(x)]^{r+1} /\left[q_{0}(r+1)\right]\right) \int_{x_{0}}^{x}\left|h^{\prime}(s)\right| d s+|K| / q_{0}\right.
$$

and dividing by $[M(x)]^{r+1}$, we have 


$$
\begin{aligned}
{[M(x)]^{1-r} } & \leq 2 H c /\left[q_{0}(r+1)\right]+\left(2 c /\left[q_{0}(r+1)\right]\right) \int_{x_{0}}^{x}\left|h^{\prime}(s)\right| d s+|K| c /\left[q_{0}[M(x)]^{r+1}\right] \\
& \leq 2 H c /\left[q_{0}(r+1)\right]+\left(2 c /\left[q_{0}(r+1)\right]\right) \int_{x_{0}}^{x}\left|h^{\prime}(s)\right| d s+|K| c /\left[q_{0} k^{r+1}\right] .
\end{aligned}
$$

Recalling assumption 5, the right-hand side is bounded by a constant for all $x \in\left[x_{0}, \infty\right)$, therefore $[M(x)]^{1-r}$ is bounded on $\left[x_{0}, \infty\right)$ and hence so is $y$.

Note 2. In (Tunç \& Ayhan, 2015), the $-y^{\prime}$ term necessitated an assumption on $y^{\prime}$, which we chose not to make as a matter of personal taste. This method of proof goes back to Walter in 1963 (Waltman, 1963) (for the nonlinear case). Besides (Qarawani, 2012) and (Tunç \& Ayhan 2015) mentioned earlier, (Kroopnick 1999), (Kroopnick 2008), (Nápoles Valdes, 2013) and (Tiryaki \& Zafer, 2013) also used this approach. Other related theorems include (Athanassov, 1987), (Bonheure \& Fabry, 2009), ( Capietto, Dambrosio, \& Liu 2009), (Cecchi, Došlá, \& Marini 2007), (Cheng 2008), ( Jiang, Rao, \& Shi 2013), (Kroopnick, 2010), (Tunc, C., \& Tunç E., 2007), ( Tunç, 2010), (Tunç, 2011) and (Wang, McKee, \& Usyk, 2010).

Corollary 1 Under the same assumptions as in Theorem 1, a solution of (1) on $\left[x_{0}, \infty\right)$ has a bounded derivative.

Proof. Returning to (3) in the proof of Theorem 1, it follows that

$$
\left[y^{\prime}(x)\right]^{2} \leq 2|h(x)| \mid F\left(y(x)\left|+2 \int_{x_{0}}^{x}\right| h^{\prime}(s)|| F(y(s)|d s+| K \mid .\right.
$$

Since $y$ has been proven to be bounded on $\left[x_{0}, \infty\right)$, say by $Y$, defining $M$ as in the proof of Theorem 1 and letting $H$ be the bound for $h$, it follows that for all $x \geq x_{0}$

$$
\begin{gathered}
{\left[y^{\prime}(x)\right]^{2} \leq 2 H c[M(x)]^{r+1} /(r+1)+\left(2 c[M(x)]^{r+1} /(r+1)\right) \int_{x_{0}}^{\infty}\left|h^{\prime}(s)\right| d s+|K| \leq} \\
2 H c Y^{r+1} /(r+1)+\left[2 c Y^{r+1} /(r+1)\right] \int_{x_{0}}^{\infty}\left|h^{\prime}(s)\right| d s+|K|,
\end{gathered}
$$

the right-hand side of which is bounded.

Corollary 2 Make the same assumptions as in Theorem 1 along with the additional assumption that there exists a $p_{0}$ such that $p \geq p_{0}$ on $\left[x_{0}, \infty\right)$. Then, the derivative of a solution on of $(1)$ on $\left[x_{0}, \infty\right)$ is in $L^{2}\left(\left[x_{0}, \infty\right)\right)$.

Proof. Returning again to (3) in the proof of Theorem 1, it follows that

$$
2 \int_{x_{0}}^{x} p(s)\left[y^{\prime}(s)\right]^{2} d s \leq 2|h(x)| \mid F\left(y(x)\left|+2 \int_{x_{0}}^{x}\right| h^{\prime}(s)|| F(y(x)|d s+| K \mid .\right.
$$

Applying the bounds $Y$ for $y, H$ for $|h|$ and the definition of $M$ from the proof of Theorem 1, we have for all $x \in\left[x_{0}, \infty\right)$

$$
\begin{gathered}
\int_{x_{0}}^{x} p(s)\left[y^{\prime}(s)\right]^{2} d s \leq H c[M(x)]^{r+1} /(r+1)+\left(c[M(x)]^{r+1} /(r+1)\right) \int_{x_{0}}^{x}\left|h^{\prime}(s)\right| d s+K / 2 \\
\leq H c Y^{r+1} /(r+1)+\left[c Y^{r+1} /(r+1)\right] \int_{x_{0}}^{x}\left|h^{\prime}(s)\right| d s+K / 2,
\end{gathered}
$$

and applying the additional assumption that there exists a $p_{0}$ such that $p \geq p_{0}$ on $\left[x_{0}, \infty\right)$, we have the desired result.

Proposition 1 Make the same assumptions as Theorem 1, except do not make assumption 5. Then, any solution of (1) on $\left[x_{0}, \infty\right)$ satisfies $M(x)=O\left(\left(\int_{x_{0}}^{x}\left|h^{\prime}(s)\right| d s\right)^{1 /(1-r)}\right)$, where $M(x)=\max _{s \in\left[x_{0}, x\right]}|y(s)|$ and $O$ is big-oh.

Proof. Following the proof of Theorem 1, we get 


$$
\begin{gathered}
q_{0}[y(x)]^{2} \leq 2|h(x)| \mid F\left(y(x)\left|+2 \int_{x_{0}}^{x}\right| h^{\prime}(s)|| F(y(x)|d s+| K \mid \Longrightarrow\right. \\
{[y(x)]^{2} \leq 2\left[\left|h\left(x_{0}\right)\right|+\int_{x_{0}}^{x}\left|h^{\prime}(s)\right| d s\right] \mid F\left(y ( x ) | / q _ { 0 } + ( 2 / q _ { 0 } ) \int _ { x _ { 0 } } ^ { x } | h ^ { \prime } ( s ) | | F \left(y(x)|d s+| K \mid / q_{0} .\right.\right.}
\end{gathered}
$$

Then, similar to the proof of Theorem 1,

$$
[M(x)]^{1-r} \leq 2 c\left[\left|h\left(x_{0}\right)\right|+\int_{x_{0}}^{x}\left|h^{\prime}(s)\right| d s\right] /\left[q_{0}(r+1)\right]+\left(2 c /\left[q_{0}(r+1)\right]\right) \int_{x_{0}}^{x}\left|h^{\prime}(s)\right| d s /+|K| c /\left(q_{0} k^{r+1}\right) .
$$

We now consider extending the interval $r \in(-1,1)$ from (Qarawani, 2012), (Tunç \& Ayhan, 2015) and Theorem 1, assumption 6 . The theorem does not hold in the case $r=1$, as evidenced by the following example.

Example 1: Consider the problem

$$
\begin{gathered}
y^{\prime \prime}+y=y, \text { for } y \geq 0 \\
y(0)=0, y^{\prime}(0)=1
\end{gathered}
$$

so that $p \equiv 0, q \equiv 1, h \equiv 1, f(y) \equiv y$ and $x_{0}=0$. All of the assumptions from Theorem 1 hold except assumption 6 . Note that the solution $y(x)=x$ is unbounded on $[0, \infty)$.

The case $r \leq-1$ appears to be a little trickier than the $-1<r<1$ case. We note that none of the articles mentioned earlier that have a similar proof to Theorem 1 considered this case. We can prove the following.

Theorem 2 Consider the differential equation

$$
y^{\prime \prime}(x)+p(x) y^{\prime}(x)+q(x) y(x)=h(x) f(y(x)) \text { for } x \geq x_{0} .
$$

Assume

1) $p$ is absolutely continuous on compact subintervals of $\left[x_{0}, \infty\right)$ and $p \geq 0$ on $\left[x_{0}, \infty\right)$,

2) $q$ is absolutely continuous on compact subintervals of $\left[x_{0}, \infty\right)$ and there exists a $q_{0} \in \boldsymbol{R}$ such that $q(x) \geq q_{0}>0$ for all $x \in\left[x_{0}, \infty\right)$,

3) $q^{\prime} \leq 0$ a.e. on $\left[x_{0}, \infty\right)$,

4) $h$ is absolutely continuous on compact subintervals of $\left[x_{0}, \infty\right)$,

5) $\int_{x_{0}}^{\infty}\left|h^{\prime}(s)\right| d s<\infty$,

6) There exists an $r \in(-\infty,-1]$ and a $c \in(0, \infty)$ such that $0<|f(w)| \leq c w^{r}$ on $(0, \infty)$,

$\left.7^{\prime}\right)$ For all $w \in(1, \infty), F(w) \equiv \int_{1}^{w} f(u) d u$ exists and is finite.

Then, for any positive solution $y$ of (5) on $\left[x_{0}, \infty\right)$, either

i) For any $X>x_{0}$, there exists some $x>X$ such that $y(x)<1$, or

ii) $y$ is bounded on $\left[x_{0}, \infty\right)$.

Proof. Assume conclusion i does not hold, i.e., there is some $X>x_{0}$ such that for all $x>X$ we have $y(x) \geq 1$. For convenience, let $X=x_{0}$. Follow the proof of Theorem 1 to obtain

$$
q_{0}[y(x)]^{2} \leq 2|h(x)||F(y(x))|+2 \int_{x_{0}}^{x}\left|h^{\prime}(s)\right||F(y(s))| d s+|K| .
$$


Note that for $x \geq x_{0}$, we have $|F(y(x))| \leq \int_{1}^{y(x)}|f(u)| d u \leq \int_{1}^{y(x)} c u^{r} d u \leq c \int_{1}^{y(x)}(1 / u) d u \leq c \ln (y(x))$, in which we have made use of our assumption that conclusion i does not hold. From (6), we now have (also letting $H$ be a bound for $|h(x)|$ on $\left.\left[x_{0}, \infty\right)\right)$,

$$
q_{0}[y(x)]^{2} \leq 2 c H \ln (y(x))+2 c \int_{x_{0}}^{x}\left|h^{\prime}(s)\right| \ln (y(s)) d s+|K| .
$$

As in the proof of Theorem 1, we let $M:\left[x_{0}, \infty\right) \rightarrow \boldsymbol{R}$ be defined by $M(x)=\max _{s \in\left[x_{0}, x\right]} y(s)$. Again using the fact that conclusion i does not hold, it follows that

$$
q_{0}[M(x)]^{2} \leq 2 c H \ln (M(x))+2 c \ln (M(x)) \int_{x_{0}}^{x}\left|h^{\prime}(s)\right| d s+|K| .
$$

If $y$ is not bounded on $\left[x_{0}, \infty\right)$, there is some integer $k \geq 1$ and some $x_{1} \geq x_{0}$ such that $M\left(x_{1}\right)=e^{k}$. We can then construct an increasing sequence $\left\{x_{n}\right\}_{n=1}^{\infty}$ such that $x_{n} \rightarrow \infty$ and $M\left(x_{n}\right)=e^{k+n-1}$. From assumption $7^{\prime}$, we have

$$
q_{0}\left[M\left(x_{n}\right)\right]^{2} \leq 2 c H \ln \left(M\left(x_{n}\right)\right)+2 c \ln \left(M\left(x_{n}\right)\right) \int_{x_{0}}^{x_{n}}\left|h^{\prime}(s)\right| d s+|K| .
$$

We then have

$$
\begin{aligned}
& q_{0} e^{2(k+n-1)} \leq 2 c H \ln \left(e^{k+n-1}\right)+2 c \ln \left(e^{k+n-1}\right) \int_{x_{0}}^{x_{n}}\left|h^{\prime}(s)\right| d s+|K| \Rightarrow \\
& q_{0} e^{2(k+n-1)} \leq 2 c H(k+n-1)+2 c(k+n-1) \int_{x_{0}}^{x_{n}}\left|h^{\prime}(s)\right| d s+|K| \Rightarrow \\
& q_{0} e^{2(k+n-1)} /(k+n-1) \leq 2 c H+2 c \int_{x_{0}}^{x}\left|h^{\prime}(s)\right| d s+|K| /(k+n-1) .
\end{aligned}
$$

Letting $n \rightarrow \infty$ and recalling assumption 5, the left-hand side approaches infinity, while the right-hand side is finite - this contradicts our assumption that $y$ is not bounded on $\left[x_{0}, \infty\right)$.

Note 3. A similar theorem can be proven for a solution which is negative-valued.

If $h$ is a constant function in Theorem 2, the proof will still work even if we do not make the assumption that conclusion $\mathrm{i}$ does not occur, i.e., we find that conclusion i cannot occur if $h$ is a constant:

Corollary 3 Consider the differential equation

$$
y^{\prime \prime}(x)+p(x) y^{\prime}(x)+q(x) y(x)=f(y(x)) \text { for } x \geq x_{0} .
$$

Assume

1) $p$ is absolutely continuous on compact subintervals of $\left[x_{0}, \infty\right)$ and $p \geq 0$ on $\left[x_{0}, \infty\right)$,

2) $q$ is absolutely continuous on compact subintervals of $\left[x_{0}, \infty\right)$ and there exists a $q_{0} \in \boldsymbol{R}$ such that $q(x) \geq q_{0}>0$ for all $x \in\left[x_{0}, \infty\right)$,

3) $q^{\prime} \leq 0$ a.e. on $\left[x_{0}, \infty\right)$,

4) There exists an $r \in(-\infty,-1]$ and a $c \in(0, \infty)$ such that $0<|f(w)| \leq c w^{r}$ on $(0, \infty)$,

5) For all $v, w \in(0, \infty)$ with $v \leq w, \int_{v}^{w} f(u) d u$ exists and is finite.

Then, any positive solution $y$ of (8) is bounded.

Proof. Let $M:\left[x_{0}, \infty\right) \rightarrow \boldsymbol{R}$ be defined by $M(x)=\max _{s \in\left[x_{0}, x\right]} y(s)$. If $y$ is not bounded on $\left[x_{0}, \infty\right)$, there is some integer $k \geq 1$ and some $x_{1} \geq x_{0}$ such that $M\left(x_{1}\right)=e^{k}$. We can then construct an increasing sequence $\left\{x_{n}\right\}_{n=1}^{\infty}$ such that $x_{n} \rightarrow \infty$ and 
$M\left(x_{n}\right)=e^{k+n-1}$. Let $F(w)=\int_{e^{k}}^{w} f(u) d u$ for $w \geq e^{k}$. For any $x \geq x_{1}$, follow the proof of Theorem 1 to obtain, for each $n \in \mathbf{N}$

$$
q_{0}\left[y\left(x_{n}\right)\right]^{2} \leq 2\left|h\left(x_{n}\right)\right|\left|F\left(y\left(x_{n}\right)\right)\right|+|K| .
$$

We then have $\left|F\left(y\left(x_{n}\right)\right)\right| \leq \int_{e^{k}}^{y(x)}|f(u)| d u \leq \int_{e^{k}}^{e^{k+n-1}}|f(u)| d u \leq \int_{e^{k}}^{e^{k+n-1}} u^{r} d u \leq \int_{e^{k}}^{e^{k+n-1}} c(1 / u) d u \leq c(n-1)$. From (9), we now have (also letting $H$ be a bound for $|h(x)|$ on $\left[x_{0}, \infty\right)$ ),

$$
\begin{gathered}
q_{0}\left[y\left(x_{n}\right)\right]^{2} \leq 2 c H(n-1)+|K| \Longrightarrow \\
q_{0} e^{2 k+n-1} \leq 2 c H(n-1)+|K| .
\end{gathered}
$$

Letting $n \rightarrow \infty$ we contradict our assumption that $y$ is not bounded on $\left[x_{0}, \infty\right)$.

If $h^{\prime}$ is not identically 0 , it seems that the term $2 \int_{x_{0}}^{x}\left|h^{\prime}(s)\right| F(y(s)) d s$ in (6) might get out of control if $y$ gets too close to 0 - it seems possible that there is an example in which the solution oscillates in an unbounded manner, varying between very low values of $y$ and increasingly larger values of $y$. I would like to exhibit an example that illustrates for particular variable $h$ 's that conclusion i of Theorem 2 can actually occur, or prove that it cannot occur for any $h$, but this has eluded me to this point.

The next proposition is obvious, but we include it as a complement to the results given above.

Proposition 2 Consider the problem

$$
\begin{gathered}
y^{\prime \prime}(x)+q(x) y(x)=h(x) f(y(x)) \text { for } x \geq x_{0}, \\
y\left(x_{0}\right) \geq 0, y^{\prime}\left(x_{0}\right)>0 .
\end{gathered}
$$

If $h(x) f(w)-q(x) w>0$ for all $(x, w) \in\left[x_{0}, \infty\right) \times(0, \infty)$, then any positive solution on $\left[x_{0}, \infty\right)$ is unbounded.

Proof. Obvious, since $y^{\prime}$ must be bounded below by $y^{\prime}\left(x_{0}\right)>0$, hence $y$ grows without bound.

A simple example for Proposition 2 would be $q \equiv 0, h \equiv 1, f \equiv 1, y(0)=0$ and $y^{\prime}(0)=1$ : the resulting initial value problem has the unbounded solution $y(x)=x^{2} / 2+x$.

\section{Conclusion}

We have considered boundedness of all solutions for second order nonlinear differential equations. Theorems have been proven which cover cases of differential equations not previously considered in the literature. Examples have been given to support the results and an open question has been identified.

\section{References}

Athanassov, Z. S. (1987). Boundedness criteria for solutions of certain second order nonlinear differential equations. $J$. Math. Anal. Appl., 123, 461-479.

Bonheure, D., \& Fabry, C. (2009). Littlewood's problem for isochronous oscillators. Arch. Math. (Basel), 93, 379-388.

Capietto, A., Dambrosio, W., \& Liu, B. (2009). On the boundedness of solutions to a nonlinear singular oscillator. Z. Angew. Math. Phys., 60, 1007-1034.

Cecchi, M., Došlá, Z., \& Marini, M. (2007). On intermediate solutions and the Wronskian for half-linear differential equations. J. Math. Anal. Appl., 336, (2007), 905-918.

Cheng, C., \& Xu, J. (2008). Boundedness of solutions for a class of second-order differential equations. Nonlinear Anal., 1993-2004.

Jiang, S., Rao, F., \& Shi, Y. (2013). Boundedness of solutions for semilinear Duffing's equation with asymmetric nonlinear term. J. Inequal. Appl., 15. 
Kroopnick, A. (1999). Bounded and $\mathrm{L}^{2}$-solutions to a second order nonlinear differential equation with a square integrable forcing term. Int. J. Math. Math. Sci., 22, 569-571.

Kroopnick, A. (2008). Properties of solutions to a generalized Liénard equation with forcing term. Applied Math. ENotes, 8, 40-44.

Kroopnick, A. (2010). Bounded solutions to $x^{\prime \prime}+q(t) b(x)=f(t)$. Internat. J. Math. Ed. Sci. Tech., 41, 829-836.

Nápoles, V. J. E. (2013). A note on the qualitative behavior of some second order nonlinear equation. Appl. Appl. Math., $8,767-776$.

Qarawani, M. N. (2012). Boundedness and asymptotic behavior of solutions of a second order nonlinear differential equation. Journal of Mathematics Research, 4, 121-127.

Tiryaki, A., \& Zafer, A. (2013). Global existence and boundedness for a class of second-order nonlinear differential equations. Appl. Math. Lett., 26, 939-944.

Tunc, C., \& Tunc, E. (2007). On the asymptotic behavior of solutions of certain second-order differential equation. $J$. Franklin Inst., 344, 391-398.

Tunç, C. (2010). A note on boundedness of solutions to a class of non-autonomous differential equations of second order. Applicable Analysis and Discrete Mathematics, 4, 361-372.

Tunç, C. (2011). On the boundedness of solutions of a non-autonomous differential equation of second order. Sarajevo J. Math., 19-29.

Tunç, C., \& Ayhan, T. (2015). On the asymptotic behavior of solutions to nonlinear differential equations of the second order. Comment. Math., 55, 1-8.

Waltman, P. (1963). Some properties of solutions of $u^{\prime \prime}+a(t) f(u)=0$. Monatsh. Math., 67, 50-54.

Wang, L., McKee, R., \& Usyk, L. (2010). Continuability and boundedness of solutions to nonlinear second-order differential equations. Electron. J. Differential Equations, 2010, 12.

\section{Copyrights}

Copyright for this article is retained by the author(s), with first publication rights granted to the journal.

This is an open-access article distributed under the terms and conditions of the Creative Commons Attribution license (http://creativecommons.org/licenses/by/4.0/). 